\title{
Définir un outil simple : notion universelle ou contextuelle?
}

L'exemple des outils en os et en roches tenaces du Néolithique de Suisse occidentale

\section{François-Xavier Chauvière et Catherine Joye}

\section{(2) OpenEdition}

\section{Journals}

\section{Édition électronique}

URL : https://journals.openedition.org/artefact/9235

DOI : $10.4000 /$ artefact.9235

ISSN : 2606-9245

Éditeur :

Association Artefact. Techniques histoire et sciences humaines, Presses universitaires du Midi

Édition imprimée

Date de publication : 11 septembre 2014

Pagination : 161-172

ISBN : 978-2-271-08150-6

ISSN : 2273-0753

\section{Référence électronique}

François-Xavier Chauvière et Catherine Joye, « Définir un outil simple : notion universelle

ou contextuelle? », Artefact [En ligne], 2 | 2014, mis en ligne le 17 mai 2021, consulté le 24 août 2021

URL : http://journals.openedition.org/artefact/9235; DOI : https://doi.org/10.4000/artefact.9235

\section{(c) (†) $\ominus$}

Artefact, Techniques, histoire et sciences humaines est mise à disposition selon les termes de la Licence Creative Commons Attribution - Pas d'Utilisation Commerciale - Pas de Modification 4.0 International. 


\section{Définir un outil simple: notion universelle ou contextuelle? L'exemple des outils en os et en roches tenaces du Néolithique de Suisse occidentale}

François-Xavier CHAUVIÈRE ${ }^{1}$ Catherine JOYE ${ }^{2}$

\section{Résumé}

La notion d'outillage simple vs complexe est discutée et confrontée à des ensembles d'artefacts osseux ou en roches tenaces issus de sites palafittiques néolithiques. En abordant les outils selon plusieurs angles (niveaux de compétence, investissement technique, longueurs et fragmentation spatio-temporelle des chaînes opératoires de transformation et d'utilisation), on montre que cette notion, loin d'être universelle, doit être abordée au cas par cas, en fonction du contexte et de la composition des corpus archéologiques.

Mots-clés : chaînes opératoires, industrie osseuse, investissement technique, outillage poli.

\section{Abstract}

The concept of single vs. complex tools is discussed and confronted with sets of bone or hard stones artifacts from Neolithic pile dwelling sites. Addressing tools from several angles (skill levels, technical investment, lengths and spatio-temporal fragmentation of "chaines operatoires"), we show that this notion, far from being universal, must be addressed on a case by case basis depending on the context and the composition of the archaeological record.

Keywords: bone industry, "chaînes opératoires», polished tools, technical investment. 
La notion d'outil simple est indissociable de celle d'outil complexe. Opposées de cette façon, ces deux catégories ne devraient a priori guère générer $l^{\prime}$ «inconfort intellectuel» dans lequel se trouve pourtant régulièrement plongé le préhistorien, dès lors qu'il aborde l'étude des moyens d'action sur la matière mis en œuvre par les populations humaines sans écriture ${ }^{3}$. La dimension binaire $d^{\prime}$ une telle dichotomie reste en effet "suspecte» dans la mesure où l'on ne saurait réduire la réalité archéologique aux deux extrêmes d'une classification censée rendre compte de la diversité de l'outillage. L'existence d'un continuum ${ }^{4}$ de l'un à l'autre se pose, notamment lorsqu'il s'agit d'exprimer la variabilité des transformations physico-chimiques exercées sur la matière, que celle-ci soit osseuse, minérale ou végétale 5 .

L'outil simple est souvent caractérisé par la dimension réduite ou inexistante de son façonnage. En revanche, les modalités d'acquisition n'entrent que peu ou pas du tout dans les tentatives de définition de cette simplicité, pas plus que celles relatives à un éventuel débitage et au fonctionnement même de l'outillage. Or, considérer seulement les dernières phases des chaînes opératoires de transformation (la mise à façon) paraît inapproprié à $l^{\prime}$ heure où l'approche technologique prône l'analyse de l'ensemble du cycle opératoire dans lequel s'inscrit nécessairement $1^{\prime}$ outil ${ }^{6}$. Si l'on se résout à dépasser la notion de naturefact pour les outils simples préhistoriques ${ }^{7}$, alors il convient de reformuler les concepts mêmes de simplicité/complexité et surtout de les questionner en faisant appel à des notions autres qui permettent de qualifier et de quanti- fier les critères utilisés. On distinguera notamment:

- les degrés de technicité et d'investissement technique consentis à l'acquisition, à la fabrication et à l'utilisation d'un outil; - la longueur et la fragmentation spatio-temporelle des chaînes opératoires correspondantes;

- l'emmanchement éventuel des parties actives;

- la distribution spatiale interne aux sites archéologiques, dans lesquels on peut observer la répartition des outils simples.

Nous jalonnerons notre propos d'exemples essentiellement pris dans l'un de nos champs d'étude actuels, à savoir les outillages sur matières osseuses et en roches tenaces (lames de hache polie, principalement) issus de sites multistratifiés du Néolithique moyen et final de Suisse occidentale et des régions voisines (du Ve au III ${ }^{\mathrm{e}}$ millénaire avant J.-C.). Composantes structurantes des cultures matérielles préhistoriques, ces industries prennent place au sein de contextes de production de la subsistance qui n'excluent pas les activités de prédation (collecte, chasse et pêche).

D'une manière générale, pour le Néolithique du Plateau suisse, les notions de simplicité/complexité restent un terrain largement à défricher, du moins sous ces termes et même s'il existe effectivement certains constats à ce propos. Ainsi, pour les roches tenaces, certains auteurs ont bel et bien identifié, dans des ensembles du Néolithique de la région des Trois-Lacs (Bienne, Neuchâtel et Morat), des outils moins complètement façonnés et décrits comme tels, sans toutefois préciser questionnements et définitions ${ }^{8}$. Pour le Néolithique final, cette part de l'outillage s'impose même comme une sorte de 
marque de fabrique de la période, avec pour conclusion plus ou moins exprimée que les populations du Lüscherz et de l'Auvernier-Cordé se satisfaisaient d'outils à bon compte en priorisant l'efficacité au détriment de la facture9. D'une part, ces remarques transmettent une image pessimiste des compétences technologiques préhistoriques et, d'autre part, il n'est guère tenu compte de l'existence, dans les mêmes corpus, d'outils plus complètement travaillés ou de pièces à très forte valeur ajoutée - notamment les haches-marteaux perforées typiques de cette période.

En définitive, l'objectif de notre démarche est de proposer une réflexion contextuelle de l'outil simple/complexe, valable à une échelle chronologique et géographique précisément délimitée.

\section{En amont et en aval du façonnage : acquisition, débitage et fonctionnement d'un outil simple}

Comme nous venons de le signaler, l'outil simple est communément défini comme relevant d'un façonnage sommaire, voire inexistant. Tentons ici de le caractériser autrement en le réintégrant au sein des quatre phases obligées d'une chaîne opératoire, qui mène de l'acquisition d'une matière première jusqu'au fonctionnement et à l'abandon d'un produit fini, en passant par sa fabrication.

\section{Modalités d'acquisition}

Pour l'outillage en pierre notamment, il est généralement fait état d'une opposition marquée entre l'origine des matières premières nécessaires à la fabrication des outils simples et celle des outils complexes ${ }^{10}$. Pour les premiers, les matériaux seraient d'origine locale, alors que, pour les seconds, ils seraient de provenance allochtone. Il existe pourtant de nombreux contre-exemples à cette affirmation. Ainsi, dans le Néolithique final du site de Saint-Blaise/Bains des Dames (Neuchâtel, Suisse), dendrodaté entre 3139 à
2560 av. J.-C., la serpentinite à diallage utilisée dans la fabrication des haches-marteaux est d'origine strictement locale et provient des moraines würmiennes érodées par les cours d'eau ${ }^{11}$. Cette proximité de la ressource n'implique pas pour autant une acquisition aisée. Dans ce cas précis, c'est à la rareté même des galets de taille convenable à la confection d'objets par essence complexes - nous le verrons plus loin - que se heurte l'approvisionnement (Fig. 1).

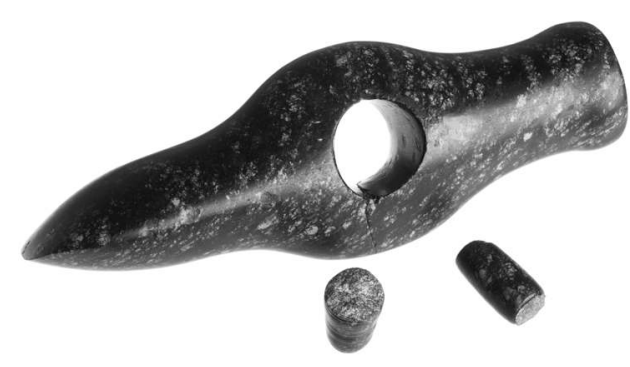

Fig. 1 - Saint-Blaise/Bains des Dames (Neuchâtel, Suisse). Auvernier-Cordé. Une hache-marteau ( $15 \mathrm{~cm}$ de longueur) et deux noyaux de perforation. Un tel objet représente le sommet des savoir-faire dans le domaine du travail des roches tenaces. (C) Laténium Marc Juillard. 
En définitive, en plus de la nature locale ou allochtone des matériaux lithiques, ce sont les modalités de l'acquisition qu'il convient de préciser, à l'aide de critères qui permettent $d$ 'interroger son caractère ciblé ou intégré (à d'autres activités) et la nature du processus dont elle relève (collecte au sens large - y compris l'extraction - le don ou l'échange). On peut y ajouter la chasse, la pêche ou l'élevage pour les matières $\mathrm{d}$ 'origine animale. Dès lors, l'acquisition du matériau dans lequel est élaboré un outil peut déjà relever d'une complexité insoupçonnée, qui doit participer à la définition du statut de l'outil.

\section{L’influence du débitage}

L'équation «façonnage sommaire = outil simple» relève à l'évidence d'un raccourci méthodologique qui ne permet pas de percevoir le déterminisme du débitage des volumes osseux ou lithiques dans la fabrication des objets.

Les pièces en os du Néolithique de Suisse occidentale, tels que les tranchants transversaux ou les pointes de type poinçon, ont ainsi longtemps été considérées comme des outils simples car les supports initiaux (des éclats) étaient interprétés comme des sous-produits de la chaîne alimentaire (orientée vers la récupération de la moelle ou la graisse des os) et ne relevaient pas d'un débitage spécifique (Fig. 2). L'analyse technique des productions sur os du Néolithique moyen de Concise-sous-Colachoz (Vaud, Suisse), dendrodaté entre 3868 et 3516 avant J.-C., a permis de proposer une autre hypothèse de travail selon laquelle la faible ampleur de la mise à façon est consécutive à un débitage élaboré ${ }^{12}$. Dès lors, il est possible de suspecter que, si les outils pris en exemples ont fait l'objet d'un façonnage restreint, c'est parce que, suite à leur segmentation longitudinale et/ou transversale, les volumes avaient déjà acquis les paramètres adéquats pour être tout à fait fonctionnels. Il n'était dès lors pas nécessaire de les façonner davantage.

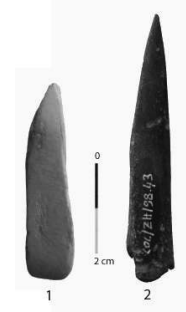

Fig. 2 - Concise sous Colachoz (Vaud, Suisse). Néolithique moyen. Exemples d'outils en os probablement emmanchés. 1. Tranchant d'extrémité sur éclat (E2 ZN170-28). 2. Pointe de type poinçon sur éclat (E5 ZH198-43). (c) F.-X. Chauvière.

Les tentatives de quantification de l'intensité du façonnage (partiel, couvrant ou total ${ }^{13}$ ) ont par ailleurs montré les possibilités de combinaisons. Ainsi, à un débitage long à mener à terme, comme le rainurage ou l'abrasion, peut être associé un façonnage réduit. À l'inverse, un débitage rapidement exécuté peut s'accompagner d'un façonnage total du volume extrait ${ }^{14}$.

\section{Modalités de fonctionnement}

Le fonctionnement ${ }^{15}$ de l'outil simple est-il lui aussi nécessairement simple? Posée de cette façon, la question est peu porteuse de sens et il vaut mieux s'interroger sur les activités techniques au sein desquelles l'outil était intégré. L'approche croisée des industries en silex 
et sur os du Néolithique final des sites de Chalain et Clairvaux (Jura français) a montré, sur la base d'études tracéologiques et expérimentales, la bipartition de ces productions entre la sphère publique et privée. La première serait réservée au monde masculin et impliquerait des activités et des outils tant complexes qu'ostentatoires. La seconde serait l'apanage des femmes et mobiliserait des outils simples au travers d'activités qui ne le seraient pas moins et qui resteraient cantonnées à la maisonnée ${ }^{16}$.

$\mathrm{Ce}$ modèle particulièrement prospectif ne peut être directement appliqué au Néolithique du Plateau suisse, au moins en l'état actuel des connaissances. Dans le cas des outils en os ou en roches tenaces, il n'existe pas, à notre connaissance, de référentiel expérimental contrôlé qui permettrait de voir à quels usages ils ont pu être destinés ${ }^{17}$. Les essais contemporains d'utilisation de lames de hache en pierre polie ont le plus souvent été dédiés à l'abattage d'arbres, une activité nécessitant des répliques d'outils à tranchant choisis parmi les plus grands ${ }^{18}$. On a par conséquent reproduit involontairement le rôle public, voire ostentatoire des plus grandes haches, tout en laissant dans l'ombre les pièces moins élaborées.

\section{La notion d'investissement technique : des critères quantifiables de la simplicité/complexité ?}

Afin de cerner le degré d'investissement consenti à la fabrication et à l'utilisation des outils, plusieurs critères doivent être pris en compte et notamment les compétences techniciennes, la longueur des chaînes opératoires, ainsi que la durée des gestes et des activités techniques.

\section{Les compétences techniciennes : représentations et opérations mentales}

Jacques Pelegrin ${ }^{19}$ opère une utile sériation sur les degrés de technicité nécessaires à l'élaboration des outillages préhistoriques, en distinguant les activités de taille des matériaux lithiques de celles des matières osseuses. Les premières nécessitent des concepts de formes géométriques, des connaissances, des capacités d'adaptation et de combinaison de ces modalités d'actions élémentaires, des capacités de programmation et d'exécution sensori-motrice de gestes de percussion adaptés. Les secondes mobilisent surtout des connaissances, des matériaux et des outils, ainsi que des modalités d'action élémentaires. Toutefois, au-delà d'une liste des compétences mises en œuvre, c'est là encore l'intensité de leur application qui reste déterminante. Une hiérarchie interne des matériaux a même été proposée pour les outillages en os et ceux en bois de cerf du Néolithique final de Chalain et Clairvau $^{20}$. Les premiers, d'élaboration rapide, seraient dédiés aux activités dites «domestiques», alors que les seconds, savamment façonnés, relèveraient des 
activités extérieures (chasse, défrichement, construction).

Les outils dits complexes ressortiraient également d'une spécialisation tant au niveau de leur fabrication que de leur utilisation. Cette dimension se marquerait spatialement par la présence de faciès d'ateliers internes aux sites, ainsi que par une forte fragmentation chrono-géographique des chaînes opératoires de fabrication/consommation ${ }^{21}$. À l'inverse, les outils simples résulteraient d'une absence de spécialisation des tâches.

\section{Courtes, moyennes ou longues: les longueurs des chaînes opératoires de transformation}

Il est également possible d'aborder la simplicité/complexité des outils préhistoriques selon la pluralité des gestes techniques, la nature (linéaire, ramifiée ou scalariforme ${ }^{22}$ ) et la longueur des chaînes opératoires qui ont présidé à l'acquisition des matériaux, à l'utilisation puis à l'abandon des produits finis. Ce séquençage de l'activité technique, en ordre de succession strict, peut se décliner en autant de phases qu'il est possible d'identifier sur chaque objet, que celui-ci soit lu en positif (diagnose de l'objet lui-même) ou en négatif (par l'intermédiaire de l'étude des déchets de fabrication, des ébauches, etc.).

Dès le début de leur étude, les corpus des outils à tranchant de Saint-Blaise/ Bains des Dames ont ainsi été considérés en fonction de l'investissement technique apporté aux objets ${ }^{23}$ (Fig. 3). En général, il a été aisé d'isoler les outils issus d'une chaîne opératoire longue (dits de type 1, comprenant une surface totalement travaillée par bouchardage avant le polissage, ou polissage intégral - «type 2») de ceux plus rapidement fabriqués (des outils sur éclats, sur fragments ou demi-galets). L'examen de cette dernière catégorie a montré qu'on pouvait en réalité y déceler deux procédures bien distinctes. L'une consiste à retoucher d'abord par taille les deux côtés du support choisi, puis d'en polir le tranchant pour obtenir une hache ou herminette à section mince, de morphologie régulière, bien que les deux faces restent largement brutes (chaîne opératoire moyenne conduisant au type 3). L'autre méthode identifiée est matérialisée par des outils plus minimalistes encore que les précédents: ce sont des éclats, dont la forme n'a même pas été reprise, un tranchant brièvement poli constituant le seul aménagement visible (type 4). De tels phasages ont également été proposés pour rendre compte des chaînes opératoires des modalités de transformation de l'os dans le Cortaillod de Concise-sous-Colachoz ${ }^{24}$ (Fig. 4).
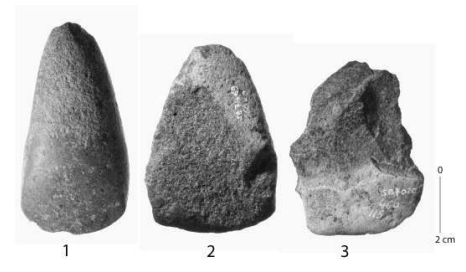

Fig. 3 - Saint-Blaise/Bains des Dames (Neuchâtel, Suisse). Auvernier-Cordé. Trois outils polis illustrant respectivement la chaîne opératoire longue (type 1), moyenne (type 3 ) et courte (type 4). Malgré leur apparente simplicité, les types 3 , comme les types 1 , étaient voués à être emmanchés. En revanche, les outils de type 4, plus expédients, ont peut-être été utilisés tenus directement en main. (C) Laténium, Marc Juillard. 


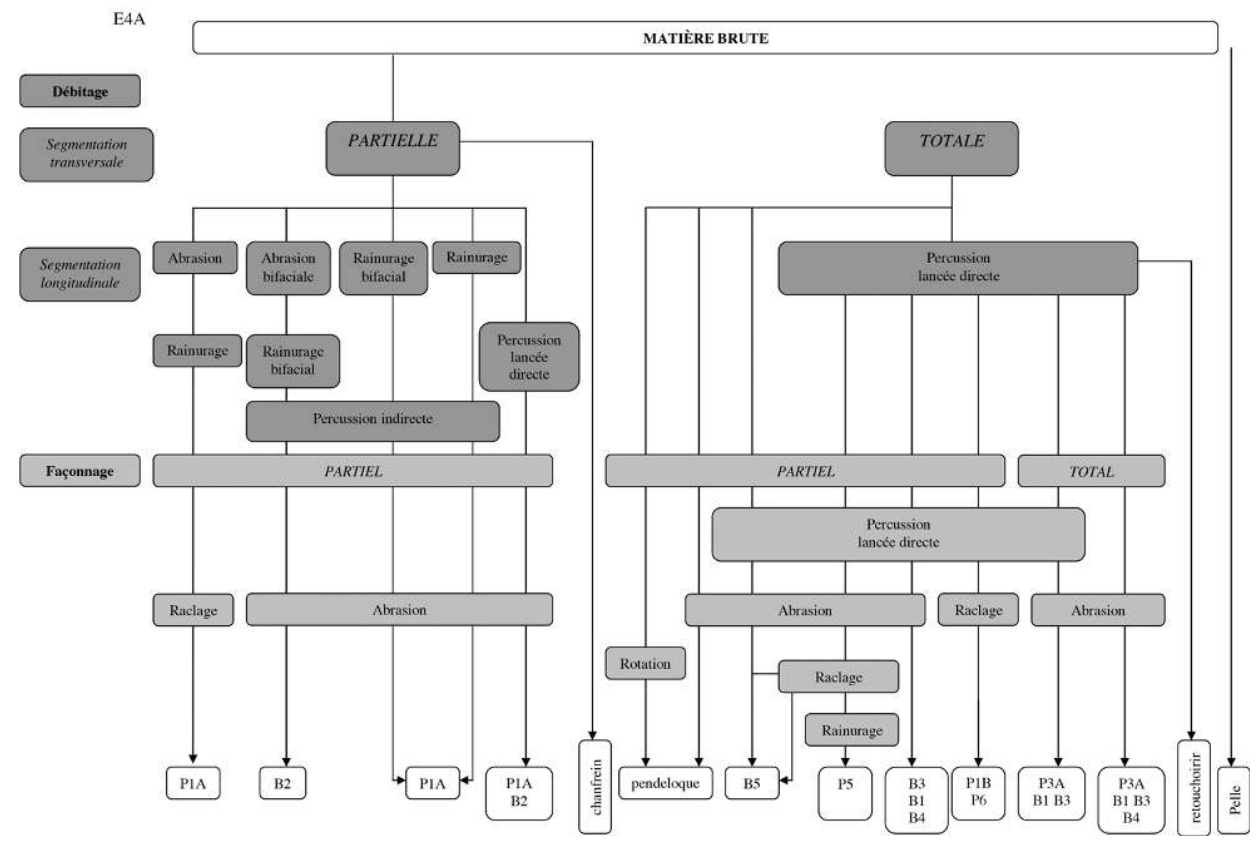

Fig. 4 - Concise sous Colachoz (Vaud, Suisse). Néolithique moyen. Exemple de chaînes opératoires de transformation de l'os (d'après Chauvière, 2010, op. cit., annexe 4d).

Les longueurs des chaînes opératoires peuvent être considérées comme des indicateurs fiables de la simplicité/complexité des outillages préhistoriques et les équations "chaîne opératoire courte = outil simple» et chaîne opératoire longue $=$ outil complexe» ont toutes les chances d'être chaque fois vérifiées. Toutefois, elles ne sauraient suffire, à elles seules, à distinguer l'outil simple du complexe dans la mesure où le critère de la longueur dépend, d'une part, d'abord du nombre de phases techniques mises en évidence (elles-mêmes soumises à la sagacité et à l'expérience de l'observateur) et, d'autre part, de la durée de ces mêmes phases techniques.

\section{Le temps court de la technique}

L'appréhension du temps long de la préhistoire a constitué l'enjeu des premières recherches sur la haute antiquité de l'homme. La recherche du temps court est une préoccupation plus récente et qui livre les clefs pour aborder la temporalité de l'acte technique ${ }^{25}$.

La durée des gestes, des phases et des activités techniques reste un critère qu'il convient de quantifier au cas par cas. Le temps de latence entre deux gestes techniques successifs n'est pas mesurable, sauf à accepter l'aide des approximations relevant du sens commun ${ }^{26}$. En soi, la quantification physique du temps nécessaire à la confection d'un objet technique n'est plus un écueil, grâce à la pratique répétée de la réplique expérimentale d'artefacts archéologiques ${ }^{27}$. 
À titre d'exemple, l'abrasion est la technique qui a été la plus usitée, tant dans la mise à façon des outils tranchants et pointus en os du Néolithique moyen de Concise-sous-Colachoz ${ }^{28}$ que dans celle des outils tranchants en roches tenaces de Saint-Blaise/Bains des Dames $^{29}$. Pourtant, on ne peut considérer comme équivalente la durée de ces deux opérations, le polissage d'une lame de hache en pierre étant beaucoup plus long (quelques dizaines d'heures) que celui d'un outil en os ou en bois de cerf (quelques minutes à une ou deux heures), quelle que soit l'épaisseur de tissu compact de ceux-ci. On le voit ici, la qualification ne peut s'abstenir d'une démarche quantitative.

\section{La partie pour le tout: le cas des outils emmanchés}

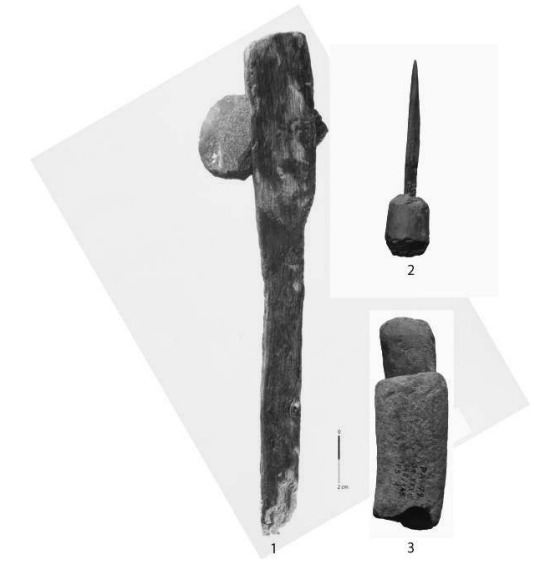

Fig. 5 - Exemples d'outils néolithiques emmanchés. 1 - Lame de hache de type 3 emmanchée (SaintBlaise/Bains des Dames. SB-BD-761. (C) Laténium, Marc Juillard); 2 et 3 - Tranchant d'extrémité en os et pointe emmanchés (Portalban, Fribourg; d'après Ramseyer, 1985).

L'archéologie préhistorique est un art $\mathrm{du}$ fragment. Les objets cassés, incomplets, sont le lot quotidien du préhistorien qui doit sans cesse restituer l'organicité d'un outil dans toute l'intégralité physique de celui-ci. Par rapport aux sites terrestres, les gisements en milieu humide de Suisse occidentale présentent, entre autres, l'exceptionnel avantage de conserver les connexions fonctionnelles entre les parties actives des outils et leur manche en bois végétal ou animal. Ils offrent ici une documentation de premier plan pour statuer de manière définitive sur les outils simples (formés d'un seul élément) et les outils complexes (composés d'éléments différents). Ils permettent ainsi de réintégrer dans la sphère des instruments ${ }^{30}$ des éléments interprétés ailleurs comme simples en l'absence physique du système d'emmanchement.

Ainsi, sans les exemplaires de haches complètes découvertes dans certains sites palafittiques, il n'aurait pas été possible de suspecter, dans la série d'objets à tranchants en roches tenaces de SaintBlaise/Bains des Dames, l'emmanchement des outils du type 3 , issus d'une chaîne opératoire de longueur moyenne et considérés comme simples au vu de leur façonnage limité (Fig. 5). Pourtant, ce type de lame constitue en définitive la partie active d'un objet nettement plus complexe: la hache ou l'herminette, variante moins sophistiquée des haches du type 1, mais composée comme elles d'au moins un manche en bois et éventuellement d'un intermédiaire, la gaine en bois de cerf ${ }^{31}$. 
Il en va de même des tranchants ou des pointes en os peu façonnés, et retrouvés emmanchés dans les sites néolithiques de Muntilier Platzbunden et Portalban (Fribourg, Suisse) $)^{32}$. Ces objets sont aussi à l'évidence des outils relativement complexes. De prime abord, sans la présence de l'emmanchement, ils émargeraient à la catégorie des outils simples ${ }^{33}$.

\section{Les données de l'analyse spatiale}

L'approche de la distribution spatiale des outillages préhistoriques peut se faire à deux échelles.

L'une rend compte de la fragmentation géographique - et donc temporelle - des chaînes opératoires de transformation et d'utilisation. Dans le cas d'un outil simple, la totalité des phases techniques qui régissent sa durée de vie, de l'acquisition du matériau jusqu'à l'abandon de la pièce finie, est censée s'être déroulée dans un seul et même lieu. C'est effectivement le cas à Saint-Blaise/Bains des Dames pour les outils à tranchants en roches tenaces. À l'inverse, la chaîne opératoire de transformation/consommation d'un outil plus complexe serait fortement fragmentée dans l'espace et donc dans le temps. On peut toutefois aisément trouver des contre-exemples à cette dernière adéquation. Toujours à SaintBlaise/Bains des Dames, la fabrication et l'utilisation des haches-marteaux de l'Auvernier-Cordé, objets situés au sommet des savoir-faire et de l'investissement technique, ont été intégralement mises en œuvre sur place et dans chaque maison, comme le démontre la répartition planimétrique des éclats de mise en forme des blocs, des ébauches, des préformes, des noyaux de perforation et des objets cassés en cours d'élabora- tion $^{34}$. Cet ensemble de faits témoigne également de l'absence de spécialisation de la fabrication d'un objet pourtant très investi techniquement ${ }^{35}$. Plus loin dans le temps, dans les ensembles du Protomagdalénien du Blot (Cerzat, HauteLoire, vers 22000 av. J.-C.), par exemple, l'étude technique des industries sur matières osseuses incite à envisager l'emport, hors du gisement, d'objets généralement considérés comme des outils simples, à savoir des pointes sur extrémité $\mathrm{d}^{\prime}$ ulna de renne mises à façon in situ pour une utilisation différée ${ }^{36}$.

L'autre focale privilégie la dimension interne des gisements archéologiques. Elle est en lien avec l'approche dendrochronologique des pieux des sites palafittiques d'Europe occidentale et centrale qui a ouvert la voie à une archéologie de la maisonnée préhistorique, dans la mesure où il est possible de délimiter le plan et la durée d'occupation des différentes constructions, généralement considérées comme des unités d'habitation. Ainsi, à Saint-Blaise/Bains des Dames, l'analyse de la distribution spatiale des différents types techniques d'outils en roches tenaces a permis certains constats. L'emplacement des outils semble varier en fonction de la longueur des chaînes opératoires desquelles ils sont issus. Les moins investis techniquement (de types 
3 et 4 ) se retrouvent ainsi souvent en position de rejet, dans les dépotoirs attenants aux maisons. À l'inverse, les outils plus élaborés (de type 1) sont préférentiellement localisés dans les unités d'habitation ${ }^{37}$.

\section{Conclusion}

S'interroger sur les notions de simple vs complexe n'est pas fortuit. Cette dichotomie renvoie à d'autres débats qui concernent la dimension individuelle ou universelle de concepts utilisés notamment en histoire ou en anthropo$\operatorname{logie}^{38}$. Inclure d'emblée l'objet d'étude (en l'occurrence ici les outils préhistoriques) dans les limites mouvantes d'une bipolarité nécessaire procède toutefois, à notre sens, d'un présupposé et d'une logique proche de celle de la "pensée sauvage $\mathrm{e}^{39}{ }$. En outre, ce positionnement méthodologique relève tout autant de la tradition analogique (mâle/femelle; nature/culture; cru/cuit, etc.) que d'une vision fantasmée de la réalité archéologique $^{40}$. Actuellement, les champs d'investigation de l'ensemble des sciences humaines tendent à brouiller, voire à effacer ces frontières que l'on pensait pourtant définitivement établies.

\section{Remerciements}

Nous exprimons toute notre gratitude à Sophie A. de Beaune qui a sollicité notre contribution à ce dossier. Sa confiance et ses conseils avisés ont été d'une aide plus que précieuse dans la rédaction de cet article.

Nous adressons nos plus vifs remerciements à Rémi Martineau et Thierry

Bonnot pour leur relecture critique et constructive de cet article, qu'ils ont ainsi contribué à améliorer, ainsi qu'à Sonia Wüthrich, archéologue cantonale de Neuchâtel, pour nous avoir permis d'utiliser les clichés du matériel de Saint-

Blaise/Bains des Dames. à opposer aussi diamétralement les outils préhistoriques. Dans leur acception la plus commune, la notion de simple/ complexe ne semble en effet pas pouvoir rendre compte de la totalité des situations archéologiques rencontrées. Selon nous, il faut renoncer définitivement à la portée universelle de définitions qu'il convient en réalité de formuler pour chaque contexte analysé, en fonction de chaque ensemble de mobilier, matière ou corpus archéologique. Technicité, investissement technique, longueurs des chaînes opératoires peuvent traduire de manière sensible et contrôlée la variabilité d'outils qui, simples ou complexes, gardent en commun d'être des moyens efficaces d'action sur la matière. 


\section{Notes}

1. François-Xavier Chauvière est archéologue à l'Office du patrimoine et de l'archéologie de Neuchâtel (Suisse), où il dirige les éditions Archéologie neuchâteloise. Il enseigne l'archéologie préhistorique à l'université Jean Moulin Lyon 3. Auteur de nombreux articles sur les industries osseuses et la parure préhistorique, il co-dirige la fouille du site Paléolithique supérieur du Petit Cloup Barrat (Lot). Il a entre autres dirigé La grotte du Bichon, un site préhistorique des montagnes neuchâteloises, éditions Archéologie neuchâteloise. Contact: francois-xavier.chauviere@ne.ch; https://univ-lyon3. academia.edu/Fran\%C3\%A7oisXavierChauviere.

2. Catherine Joye est archéologue à l'Office du patrimoine et de l'archéologie de Neuchâtel (Suisse). Spécialiste des outillages en pierre polie des sites palafittiques, elle est l'auteure de Hauterive-Champréveyres. Le village du Cortaillod classique: étude de l'outillage en roches polies et SaintBlaise/Bains des Dames. Haches et haches-marteaux en roches tenaces. De l'utilitaire à l'affichage social au Néolithique final, parus aux éditions Archéologie neuchâteloise. Contact: catherine-joye@ne.ch; https://unine.academia.edu/CatherineJoye.

3. Laurence Astruc, Françoise Bon, Vanessa Léa, Pierre-Yves Milcent et Sylvie Philibert (dir.), Normes techniques et pratiques sociales. De la simplicité des outillages pré- et protohistoriques. Actes des XXVI rencontres internationales d'archéologie et d'histoire d'Antibes, 20-22 octobre 2005, Antibes, APDCA, 2006; Yolaine Maigrot et Hugues Plisson, «Simplicité et complexité en archéologie préhistorique. Le patchwork conceptuel ou les tentations de l'ethnocentrisme», dans Laurence Astruc et al., 2006, op. cit., p. 25-33.

4. Au sens de Alice M. Choyke, «The bone manufacture continuum», Anthropozoologica, $\mathrm{n}^{\circ} 25-26,1997$, p. 65-72.

5. Daniel Boucard, Dictionnaire des outils et instruments pour la plupart des métiers, Seld, Jean-Cyrille Godefroy, 2006.

6. Sophie A. de Beaune, «De la pierre à l'os: ou comment reconstituer des chaînes techniques opératoires impliquant l'os et la pierre", dans Michèle Julien et al. (dir.), Préhistoire d'os. Recueil d'études sur l'industrie osseuse préhistorique offert à Henriette Camps-Fabrer, Aix-en-Provence, Publications de 1'Université de Provence, 1999, p. 151-158; André Leroi-Gourhan, Évolution et techniques. L'homme et la matière, Paris, Albin Michel, Coll. Sciences d'aujourd'hui, 1971.

7. Sophie A. de Beaune, ce numéro.

8. Catherine Buret, L'industrie de la pierre polie du Néolithique moyen et récent à Auvernier, canton de Neuchâtel (Suisse), thèse de doctorat multicopiée, 1983, Université Paris X Nanterre; Christoph Willms, Die neolitischen Ufersiedlungen von Twann, 9. Die Felsgestei-narte-fakte der Cortaillod-Schichten, Berne, Staatlicher Lehrmittelverlag, 1980.

9. Michel Egloff, 1973, "Contribution à la connaissance du Néolithique final de Saint-Blaise (lac de Neuchâtel, Suisse)», dans L'homme, hier et aujourd'hui. Recueil d'études en hommage à André Leroi-Gourhan, Paris, Cujas, 1973, p. 521-532 ; François Schifferdecker, Schibler Jörg, «Auvernier $\mathrm{NE}$ », dans Werner E. Stöckli et al. (dir.), La Suisse du Paléolithique à l'aube du Moyen Âge. De l'Homme de Néandertal à Charlemagne, 2. Néolithique, Bâle, Société suisse de préhistoire et d'archéologie (SPM, II), 1995, p. 303.

10. Sophie A. de Beaune, ce numéro.

11. Catherine Joye, «Les haches perforées de Saint-Blaise "Bains des Dames" (Neuchâtel, Suisse). Chaîne opératoire et analyse spatiale: une autre lecture», Bulletin de la Société préhistorique française, vol. 109, n², 2012, p. 299-310; Catherine Joye, Saint-Blaise/Bains des Dames, 5. Haches et haches-marteaux en roches tenaces. De l'utilitaire à l'affichage social au Néolithique final, Neuchâtel, Office du patrimoine et de l'archéologie, Archéologie neuchâteloise, 52, 2013.

12. François-Xavier Chauvière, «Le travail de l'os, de l'ivoire et de la dent: les données du Néolithique moyen", dans Ariane Wiiniger et al., La station lacustre de Concise 3. Le mobilier organique et lithique du Néolithique moyen, Lausanne, Cahiers d'archéologie romande, $\mathrm{n}^{\circ} 119,2010$, p. 93-132.

13. Respectivement moins de $50 \%$, plus de $50 \%$ et $100 \%$ du volume façonné.

14. François-Xavier Chauvière, 2010, op. cit., p. 106.

15. Au sens de François Sigaut, «Un couteau ne sert pas à couper mais en coupant. Structure et fonction dans l'analyse des objets ", dans 25 ans d'études technologiques en Préhistoire, $\mathrm{X}^{e}$ rencontres internationales d'archéologie et d'histoire d'Antibes, Juan-les-Pins, APDCA, 1991, p. 21-34; Sophie A. de Beaune, Pour une archéologie du geste, Paris, CNRS Éditions, 2000.

16. Valérie Beugnier et Yolaine Maigrot, «La fonction des outillages en matières dures animales et en silex au Néolithique final. Le cas des sites littoraux des lacs de Chalain et Clairvaux (Jura, France) au $\mathrm{xxx}^{\mathrm{e}}$ siècle avant notre ère ", Bulletin de la Société préhistorique française, vol. 102, n² 2, 2005, p. 335-344.

17. Les propositions les plus récentes pour le fonctionnement des objets en os, riches de perspectives, relèvent d'une démarche encore 
exploratoire, cf. Justine Mayca et Maxence Bailly, «L'écorce, le liber et les pointes plates. Caractérisation tracéologique de la pérennité fonctionnelle d'un outil emblématique des stations littorales de la baie d'Auvernier (NE, Suisse)», dans Patricia C. Anderson et al., Regards croisés sur les outils liés au travail des végétaux. An interdisciplinary focus on plant-working tools, XXXIII ${ }^{e}$ rencontres internationales d'archéologie et d'histoire d'Antibes, Antibes, APDCA, 2013, p. 309-321.

18. Béat Arnold, «Archéologie expérimentale: la pirogue néolithique expansée Paris-Bercy 6 et les arts du feu », dans Béat Arnold, Nicole Bauermeister et Denis Ramseyer (dir.), Archéologie plurielle. Mélanges offerts à Michel Egloff à l'occasion de son $65^{e}$ anniversaire, Neuchâtel, Service et musée cantonal d'archéologie (Archéologie neuchâteloise, 34), 2006, p. 65-77.

19. Jacques Pelegrin, «Le milieu intérieur d'André Leroi-Gourhan et l'analyse de la taille de pierre au Paléolithique», dans Françoise Audouze et Nathan Schlanger (dir.), Autour de l'homme. Contexte et actualité d'André Leroi-Gourhan, Antibes, APDCA, 2004, p. 149-162.

20. Yolaine Maigrot, «Le débitage du bois de cerf au Néolithique final à Chalain et Clairvaux (Jura, France). Approche expérimentale», dans Laurence Bourguignon, Illuminada Ortega et Marie-Chantal Frère-Sautot (dir.), Préhistoire et approche expérimentale, Montagnac, Monique Mergoil, Préhistoires n 5, 2001, p. 165-172; Yolaine Maigrot, Étude technologique et fonctionnelle de l'outillage en matières dures animales. La station 4 de Chalain (Néolithique final, Jura, France), thèse de doctorat, Université de Paris I-PanthéonSorbonne, 2003.

21. Laurence Astruc, «Approche multi-échelles de la spécialisation technique. Les industries lithiques taillées du Néolithique pré-céramique au Proche-Orient», Techniques \& Culture, $\mathrm{n}^{\circ}$ 46-47, 2005-2006, p. 79-104.

22. Jean-Michel Geneste, "Systèmes techniques de production lithique: variations technoéconomiques dans les processus de réalisation des outillages lithiques», Techniques \& Culture, $\mathrm{n}^{\circ} 17-18,1991$, p. 1-35.

23. Catherine Joye, 2013, op. cit.

24. François-Xavier Chauvière, 2010, op. cit., annexe 4 .

25. Yvette Taborin, «Du temps long au temps court», dans Jean-Pierre Mohen (dir.), Le temps de la Préhistoire, Dijon, éd. Archéologia, t. 1, 1989, p. $94-95$.

26. François-Xavier Chauvière, "Quand le technique jalonne le temps: la notion de "temps technique" en archéologie paléolithique», dans La perception $d u$ temps en préhistoire, $129^{\mathrm{e}}$ Congrès du CTHS, Besançon, 19-24 avril 2004, Bulletin de la Société préhistorique française, vol. 102, n 4, 2005, p. $755-762$.

27. Laurence Bourguignon et al., 2001, op. cit.

28. François-Xavier Chauvière, 2010, op. cit., p. 104.

29. Catherine Joye, 2013, op. cit.

30. Au sens de Bruno Jacomy, «Outil/ machine», Notions, Notionnaires, Encyclopedia Universalis, 2004, p. 744-746.

31. Catherine Joye, 2013, op. cit.

32. Denis Ramseyer, "Pièces emmanchées en os et en bois de cervidés. Découvertes néolithiques récentes du canton de Fribourg, Suisse occidentale», dans Henriette Camps-Fabrer (dir.), Industrie de l'os néolithique et de l'âge des métaux, Première réunion $d u$ groupe de travail $n^{\circ} 3$ sur l'industrie de l'os préhistorique, 3, Paris, CNRS, 1985, p. 194-211.

33. Il existe des indices macroscopiques permettant de diagnostiquer l'emmanchement sur des pièces isolées (types de fractures, états de surface de la partie emmanchée et de la partie active, etc.). Pour les outils en os, voir Chauvière, 2010, op. cit. ; Maigrot, 2003, op. cit.

34. Matthieu Honegger, Pauline de Montmollin et Catherine Joye, «Un essai sur les premières armes de guerre du Néolithique: flèches, poignards et haches de combat au nord-ouest des Alpes», dans Luc Baray, Matthieu Honegger et Marie-Hélène Diaz-Meirino (dir.), L'armement et l'image du guerrier dans les sociétés anciennes: de l'objet à la tombe, table ronde internationale, Musée de Sens, 4-5 juin 2009, Dijon, Éditions universitaires, 2001, p. 71-102; Catherine Joye, 2013, op. cit.

35. Catherine Joye, 2012, op. cit.

36. François-Xavier Chauvière et Laure Fontana, "Modalités d'exploitation des rennes du Blot (Haute-Loire): entre subsistance, technique et symbolique», dans Véronique Dujardin (dir.), Industries osseuses et parures du Solutréen au Magdalénien en Europe, Angoulême, 28-30 mars 2003, Paris, Société préhistorique française, Mémoire $\mathrm{n}^{\circ} 30,2005$, p. 137-147.

37. Catherine Joye, 2013, op. cit.

38. Par exemple, Philippe Descola, Par-delà nature et culture, Paris, Gallimard, Bibliothèque des sciences humaines, 2005.

39. Claude Levi-Strauss, La pensée sauvage, Paris, Plon, 1962.

40. Jacques Collina-Girard, «Le foret à feu: expérimentation et rumeur scientifique», L'Homme, 120, XXXI(4), 1991, p. 69-88. 